\title{
Signatures of Majorana States in Electron Transport through a Quantum Dot Coupled to a Topological Wire
}

\author{
P. STEFAŃSKI*
}

Institute of Molecular Physics of the Polish Academy of Sciences, ul. Smoluchowskiego 17, 60-179 Poznań, Poland

\begin{abstract}
We consider theoretically a system composed of a quantum dot coupled to a topological superconducting wire. The dot, being in Coulomb blockade (CB) regime is additionally coupled to the normal leads. The topological wire hosts Majorana states, which, as we show, characteristically modifies conductance through the dot. An unpaired Majorana state in the wire causes a unique temperature dependence of zero bias conductance vs. gate voltage. It decreases in-between $\mathrm{CB}$ peaks and on the sides of the peaks from the plateau at $\sim e^{2} / 2 h$ when temperature increases. At the same time conductance increases at the CB peak positions. It is accompanied by zero bias anomaly in differential conductance. For finite overlap of Majorana states in the wire the zero bias anomaly disappears. Instead, two characteristic Fano resonances of opposite symmetry appear, positioned mirror-symmetrically with respect to zero bias.
\end{abstract}

DOI: 10.12693 /APhysPolA.127.198

PACS: 73.63.-b, 73.23.Hk, 73.21.La, 74.78.Na

\section{Introduction}

Majorana fermions, exotic quasiparticles which are their own antiparticles, were proposed by E. Majorana in the context of particle physics [1]. They are exciting due to both their fundamental properties and potential application in topologically robust quantum computing [2]. The most promising scalable system in solid state appears to be a one dimensional $p$-type superconducting wire, originating from the idea proposed by Fu and Kane [3]. This type of superconductivity is realized in a system of a semiconductor nanowire with Rashba interaction, placed in proximity to usual s-type superconductor, both subjected to an external magnetic field [4]. The first promising experimental realization was demonstrated by Mourik et.al. [5]. To make quantum computing on Majorana states feasible one needs methods for their unambiguous identification. One of them is measurement of transport characteristics of a quantum dot (QD); in Kondo regime [6] or effectively noninteracting [7], attached to one end of a topological superconducting wire. We assume that the dot is in Coulomb blockade (CB) regime and show that a simple transport measurement through the dot is sufficient to distinguish the presence of Majorana bound states in the wire. More sophisticated conditions like Kondo regime of QD or/and its symmetric coupling to the leads are not necessary. A peculiar temperature dependence of QD zero bias conductance vs. gate voltage complemented with its differential conductance vs. bias gives a sufficient information on the existence of Majorana states in the wire.

\section{The model and theoretical approach}

The schematic of the device considered is shown in Fig. 1. The total Hamiltonian has the form $H=$

\footnotetext{
*e-mail: piotrs@ifmpan.poznan.pl
}

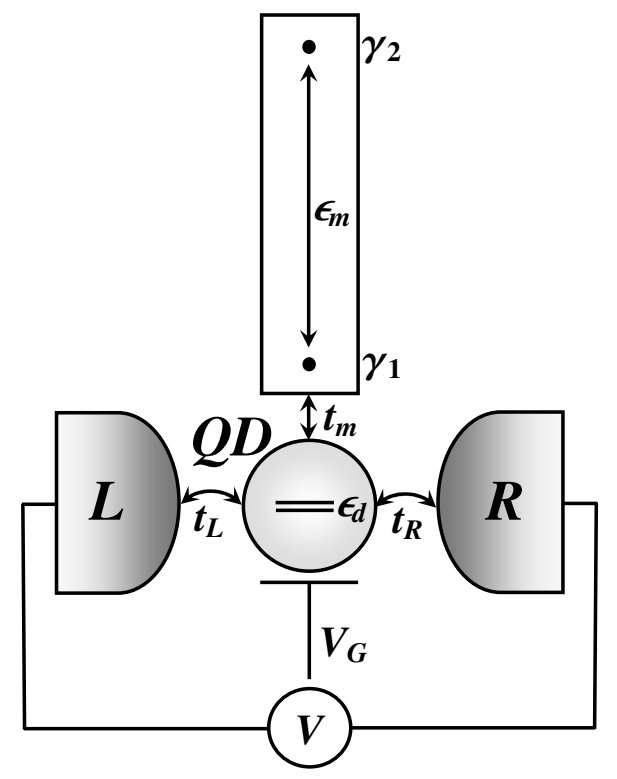

Fig. 1. Schematic of the device setup. Quantum dot (QD) is coupled to a topological superconducting wire made of semiconductor wire with large spin-orbit interaction in proximity to s-wave superconductor, both subjected to external Zeeman field.

$H_{Q D-E}+H_{Q D-M S}$, where the Hamiltonian of the QD coupled to the electrodes is as follows:

$$
\begin{aligned}
& H_{Q D-E}=\varepsilon_{d} d_{\sigma}^{\dagger} d_{\sigma}+U n_{\sigma} n_{\bar{\sigma}} \\
& +\sum_{k, \sigma, \alpha=L, R}\left[t_{\alpha} c_{k \alpha, \sigma}^{\dagger} d_{\sigma}+\text { h.c. }\right] \\
& +\sum_{k, \sigma, \alpha=L, R} \varepsilon_{k \alpha, \sigma} c_{k \alpha, \sigma}^{\dagger} c_{k \alpha, \sigma} .
\end{aligned}
$$

Operator $d_{\sigma}^{\dagger}$ creates an electron with spin $\sigma$ on the QD level $\varepsilon_{d}$. $U$ is Coulomb repulsion between dot's electron occupancies with opposite spins: $n_{\sigma}=d_{\sigma}^{\dagger} d_{\sigma}$, and $t_{\alpha}$ 
represents hopping between the dot and lead $\alpha=L, R$. Finally, $c_{k \alpha, \sigma}^{\dagger}$ creates electron with momentum $|k|$, spin $\sigma$ and energy $\varepsilon_{k \alpha}$ in the lead $\alpha$. The QD's level $\varepsilon_{d}$ can be shifted by gate voltage $V_{G}$ with respect to Fermi level, positioned at zero energy, $\varepsilon_{F}=0$. We assume that the critical Zeeman field needed to induce $p$-wave superconductivity, which is $\sim 0.15 \mathrm{~T}[5]$, is low enough not to induce observable Zeeman splitting of the dot's active level due to a small $g$-factor in quantum dots [8]. For $g=-0.44$ it introduces negligible Zeeman splitting $\sim 3.8 \mu \mathrm{eV}$. The dot is additionally side-coupled to Majorana bound state (MBS) by the hopping $t_{m}$ :

$$
H_{Q D-M S}=t_{m}\left(d_{\downarrow}^{\dagger} \gamma_{1}+\gamma_{1} d_{\downarrow}\right)+2 \mathrm{i} \varepsilon_{m} \gamma_{1} \gamma_{2} \text {. }
$$

We assume that spin-down sector only of the dot is coupled to the MS due to its helical properties [9]. $\varepsilon_{m} \sim$ $\mathrm{e}^{-L / \xi}$ represents the coupling between two Majorana states, where $L$ is the wire length and $\xi$ is the superconducting coherence length. The Majorana operators can be expressed in terms of ordinary fermionic operators: $\gamma_{1}=\left(f+f^{\dagger}\right) / \sqrt{2}$ and $\gamma_{2}=-\mathrm{i}\left(f-f^{\dagger}\right) / \sqrt{2}$.

Our goal is to calculate QD retarded Green's function $G_{\sigma}^{r}\left(t-t^{\prime}\right)=-\mathrm{i} \theta\left(t-t^{\prime}\right)\left\langle d_{\sigma}(t) d_{\sigma}^{\dagger}\left(t^{\prime}\right)+d_{\sigma}^{\dagger}\left(t^{\prime}\right) d_{\sigma}(t)\right\rangle$. In order to do this we first rewrite $H_{Q D-M S}$ in terms of usual fermionic operators and then apply equation of motion (EOM) technique for Green's functions in Hubbard approximation [10], see also [11].

We have further introduced the notation $\tilde{t}_{m}=t_{m} / \sqrt{2}$ and $\tilde{\varepsilon}_{m}=2 \varepsilon_{m}$. In the wide band limit of the density of states in the leads $\rho_{0}\left(\equiv \rho_{L}=\rho_{R}\right)$ we obtain: $\sum_{k, \alpha}\left|t_{\alpha}\right|^{2} /\left(\omega \mp \varepsilon_{k \alpha}\right)=-(i / 2) \sum_{\alpha} \Gamma_{\alpha} \equiv-\mathrm{i} \Gamma$, where $\Gamma_{\alpha}=2 \pi\left|t_{\alpha}\right|^{2} \rho_{0}$ is the broadening gained by QD level coupled to lead $\alpha$. The obtained dot's Green's function, $G_{d, \downarrow}^{r}(\omega) \equiv\left\langle\left\langle d_{\downarrow} ; d_{\downarrow}^{\dagger}\right\rangle\right\rangle_{\omega}$ has the form:

$$
\begin{aligned}
& G_{d, \downarrow}^{r}(\omega)=\frac{X_{-}-M-\frac{M^{2} B}{C_{+}-M H_{+}+\mathrm{i} \Gamma X_{+}}}{C_{-}-M H_{-}+\mathrm{i} \Gamma X_{-}+\frac{M^{2} A}{C_{+}-M H_{+}+\mathrm{i} \Gamma X_{+}}}, \\
& X_{ \pm}=\omega \pm \varepsilon_{d} \pm U\left(1-\left\langle n_{\uparrow}\right\rangle\right), \\
& C_{ \pm}=\left(\omega \pm \varepsilon_{d}\right)\left(\omega \pm \varepsilon_{d} \pm U\right), \\
& H_{ \pm}=2\left(\omega \pm \varepsilon_{d}\right) \pm U+\mathrm{i} \Gamma, \\
& A=2\left(\omega+\varepsilon_{d}\right)^{2}+\Gamma^{2}, \\
& B=2 \varepsilon_{d}+U+\mathrm{i} \Gamma, \\
& M=\frac{2 \tilde{t}_{m} \omega}{\omega^{2}-\varepsilon_{m}^{2}} .
\end{aligned}
$$

Upon substitution into Eq. (3) $t_{m}=0$ and exchange of spin indices we obtain familiar Hubbard Green's function for spin-up sector, uncoupled from the topological wire. It has two charge peak structure placed at Hubbard resonances $\varepsilon_{d \uparrow}^{I} \equiv \varepsilon_{d \uparrow}$ and $\varepsilon_{d \uparrow}^{I I} \equiv \varepsilon_{d \uparrow}+U$.

QD occupancies for spin-up $\left\langle n_{\uparrow}\right\rangle$ and spin-down $\left\langle n_{\downarrow}\right\rangle$ have been calculated selfconsistently and the current has been calculated within Landauer formalism; for the methods applied see [11].

\section{Numerical results and discussion}

In numerical calculations we took a constant value of density of states in the leads: $\rho_{0}=1 / 2 D$ with width $2 D$. The quantity $D$ is taken as a unit. The ratio $U / \Gamma=20$ $\left(\Gamma \equiv \Gamma_{L}=\Gamma_{R}\right)$ has been taken large in the paper. From the experimental point of view it prevents the formation of the strongly correlated state in the dot and Kondo effect in the investigated temperature range.

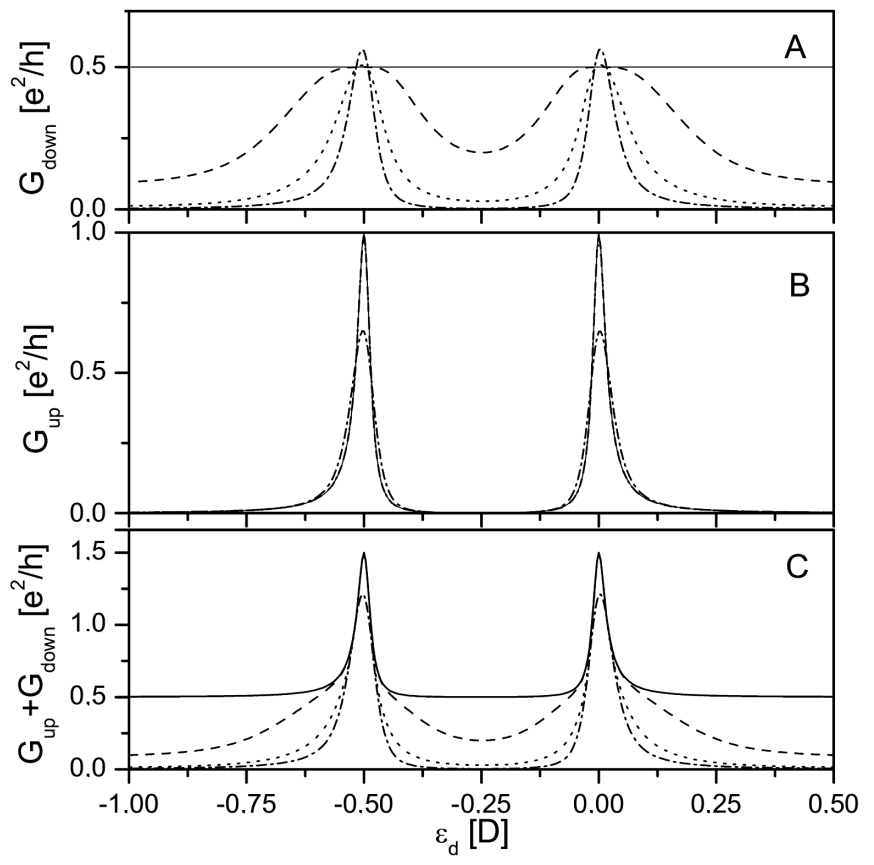

Fig. 2. Zero bias conductance vs. QD level position for spin-up (A), spin-down (B) sectors and the sum (C). The calculations are performed for various temperatures: $T=10^{-4}-$ dashed line, $T=10^{-3}-$ dotted line and $T=10^{-2}-$ dash-dotted line. Conductance for $T=0$ - solid line has also been calculated for comparison. Other calculations parameters are $t_{m}=0.01$, $\varepsilon_{m}=0, U=0.5, \Gamma_{L}=\Gamma_{R}=0.05 U$.

Zero bias conductance and its spin components vs. QD level position for $\varepsilon_{m}=0$ and various temperatures is shown in Fig. (2). In $T=0$ the conductance in spindown sector $G_{\downarrow}=e^{2} / 2 h$ for symmetric coupling, independently of the position of the dot's level. It is caused by MBS located at Fermi energy, which conducts half of a fermion. When temperature increases the spindown conductance begins to depart from the value of half conductance quantum toward lower values. On the contrary, at the same time it begins to increase at the Coulomb blockade peak positions. It causes a characteristic behavior of the total conductance. It cannot be mixed with Kondo physics [12], where the conductance decreases in-between CB peaks but increases on the sides of the peaks. It has also opposite effect as compared to usual temperature enhancement of the background conductance. Importantly, this peculiar behavior of the conductance is robust to the asymmetry of the dot's coupling to the leads. For instance for extremely asymmetric 
arrangement $\Gamma_{R}=0.1 \Gamma_{L}$ it has exactly the same behavior as shown in Fig. (2) with conductance values scaled down. The constant plateau of $G_{\downarrow}$ in $T=0$ now assumes the value $\sim 0.16\left(e^{2} / h\right)$, as compared to $0.5\left(e^{2} / h\right)$ for symmetric coupling.

For finite overlap, $\varepsilon_{m} \neq 0$, between MBS in the wire they form a Dirac fermion $f$. The spin-down conductance is practically undistinguishable from spin-up conductance and displays two Coulomb blockade peaks. Thus, zero bias conductance measurements are in this case not sufficient for spotting Majorana states. A measurement of the bias dependence of differential conductance gives a complementary information on the existence of Majorana bound states. In Fig. (3) a representative differential

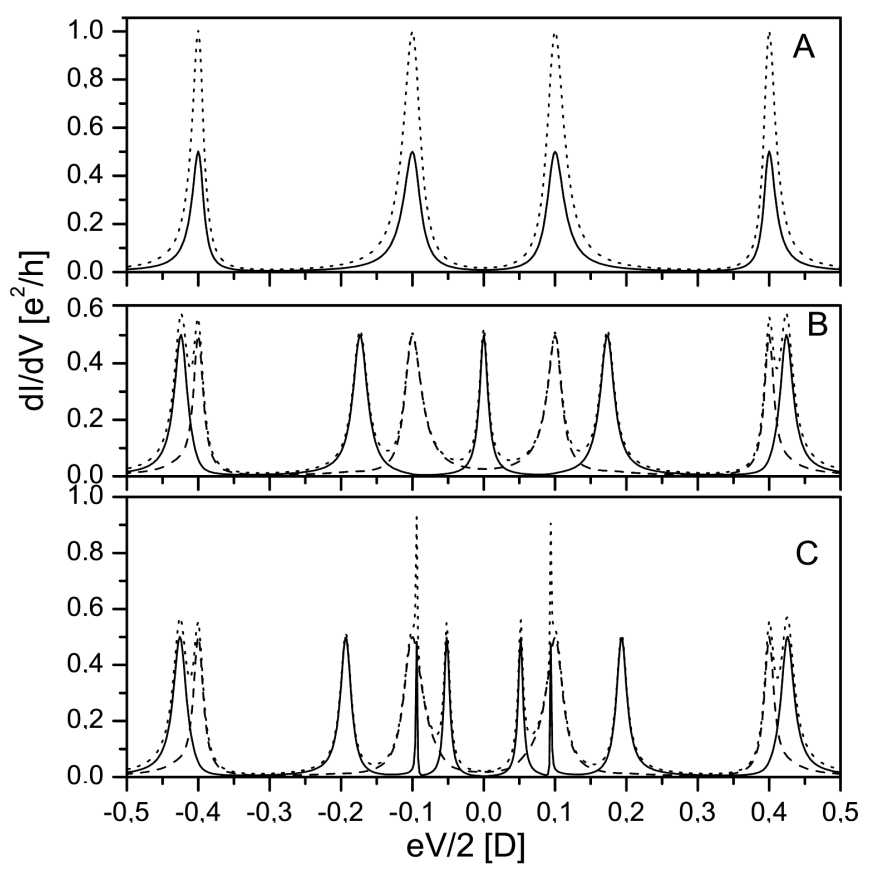

Fig. 3. Differential conductance vs. bias voltage for spin-up (dashed), spin-down (solid) and the sum (dotted) for $\varepsilon_{d}=-0.1$. Panel (A) is for $t_{m}=0$, panel (B) is for $t_{m}=0.1$ and $\varepsilon_{m}=0$, panel $(\mathrm{C})$ is for $t_{m}=0.1$ and $\varepsilon_{m}=0.05$. The calculations are performed in temperature $T=10^{-4}$ and $U=0.5, \Gamma_{L}=\Gamma_{R}=0.05 U$

conductance vs. bias for $\varepsilon_{d}=-0.1$ is presented. For the dot coupled to the leads only, shown in panel (A), differential conductance exhibits four peaks. They develop each time when one of the chemical potential of the leads, $\mu_{\alpha}$, comes into resonance with one of the Hubbard peaks. Starting from negative bias we have the sequence of resonances: for $\mathrm{eV} / 2 \sim-0.4 \mu_{L}=\varepsilon_{d}^{I I}$, for $\mathrm{eV} / 2 \sim-0.1$ $\mu_{R}=\varepsilon_{d}^{I}, e V / 2 \sim 0.1 \mu_{L}=\varepsilon_{d}^{I}$ and $e V / 2 \sim 0.4 \mu_{R}=\varepsilon_{d}^{I I}$. In panel (B) the case when QD is hybridized with unpaired MBS is shown. The presence of Majorana bound state is indicated by zero bias peak. Also double peaked structures can be observed, positioned in mirror symmetry with respect to $e V=0$ line. For $e V / 2 \sim-0.4$ two peaks appear when $\mu_{L}=\varepsilon_{d \downarrow}^{I I}$ and $\mu_{L}=\varepsilon_{d \uparrow}^{I I}$. Similarly two peaked structure can be observed for $\mathrm{eV} / 2=0.4$, where $\mu_{R}=\varepsilon_{d \uparrow}^{I I}$ and $\mu_{R}=\varepsilon_{d \downarrow}^{I I}$. They are caused by the spin selective hybridization of the dot to Majorana state $[6,9]$. For $\varepsilon_{d}=-0.1$ the first Hubbard peak is close to the Fermi level and in spin-down sector it gets split into two peaks at $\omega \sim \mp \sqrt{2} t_{m}$ due to hybridization with MBS. At $e V / 2 \sim-0.2 \mu_{L}$ becomes aligned with the peak at $\omega \sim \sqrt{2} t_{m}$ and a the same time $\mu_{R}$ is in resonance with the peak at $\omega \sim-\sqrt{2} t_{m}$. At the bias $\mathrm{eV} / 2 \sim 0.2$ we have opposite chemical potentials alignment. As a result two peaks of equal height appear in the differential conductance at $e V / 2 \sim \mp 0.2$. Conductance peaks in spin-up sector located at $e V / 2 \sim \mp 0.1$ appear when $\mu_{R}=\varepsilon_{d \uparrow}^{I}$ and $\mu_{L}=\varepsilon_{d \uparrow}^{I}$, respectively.

Panel (C) displays the situation for $\varepsilon_{m} \neq 0$. In this case the zero bias Majorana peak disappears. Instead two characteristic Fano resonances [13] develop of opposite asymmetry, located at $\mathrm{eV} / 2 \sim \mp 0.1$ mirror symmetrically with respect to $e V=0$ line. They arise as a result of quantum interference between split hubbard resonance in spectral density, located at $\omega \sim \mp \sqrt{2} t_{m}$, with fermionic $f$ level positioned at $\omega=\mp 2 t_{m}$. The picture does not change qualitatively for asymmetric coupling, there is a downward rescaling only of the conductance values.

\section{Acknowledgments}

This work has been supported by the National Science Centre under the contract DEC-2012/05/B/ST3/03208.

\section{References}

[1] E. Majorana, Nuovo Cimento 5, 171 (1937).

[2] A. Stern, N.H. Lindner, Science 339, 1179 (2013).

[3] L. Fu, C.L. Kane, Phys. Rev. Lett. 100, 096407 (2008).

[4] T.D. Stanescu, S. Tewari, J. Phys. Condens. Matt. 25, 233201 (2013).

[5] V. Mourik, K. Zuo, S.M. Vrolov, S.R. Plissard, E.P.A.M. Bakkers, L.P. Kouwenhoven, Science $\mathbf{3 3 6}$, 1003 (2012)

[6] M. Lee, J.S. Lim, R. Lopez, Phys Rev. B 87, 241402(R) (2013).

[7] D.E. Liu, H.U. Baranger, Phys Rev. B 84, 201308(R) (2011).

[8] L.P. Kouvenhoven, D.G. Austing, S. Tarucha, Rep Prog. Phys. 64, 701 (2001).

[9] J.J. He, T.K. Ng, P.A. Lee, K.T. Law, Phys. Rev. Lett. 112, 037001 (2014).

[10] A.C. Hewson, 10.1103/PhysRev.144.420Phys. Rev. 144, 420 (1966).

[11] P. Stefański, Phys. Rev. B 79, 0859312 (2009).

[12] W.G. van der Wiel, S. de Francheschi, T. Fujisawa, J.M. Elzerman, S. Tarucha, L.P. Kouwenhoven, Science 289, 2105 (2000).

[13] E.A. Miroshnichenko, S. Flach, Y.S. Kivshar, Rev. Mod. Phys. 82, 2257 (2010). 